Sáiz Manzanares, M.C. \& Valdivieso-León, L. (2020). Relación entre rendimiento académico y desarrollo de Estrategias de autorregulación en estudiantes universitarios. Revista Electrónica Interuniversitaria de Formación del Profesorado, 23(3), 49-65.

DOI: https://doi.org/10.6018/reifop.385491

\title{
Relación entre rendimiento académico y desarrollo de estrategias de autorregulación en estudiantes universitarios
}

María Consuelo Sáiz Manzanares ${ }^{1}$ y Lorena Valdivieso-León ${ }^{2}$

Universidad de Burgos ${ }^{1}$ y Universidad de Valladolid ${ }^{2}$

\section{Resumen}

En este estudio se analiza la relación entre la auto-percepción que los estudiantes tienen de su conocimiento declarativo y procedimental con el rendimiento académico. Participaron 116 estudiantes universitarios de primero y segundo curso de grado en dos estudios, uno cuasiexperimental (antes-después sin grupo control) donde la variable independiente fue la "enseñanza basada en estrategias de autorregulación" y la variable dependiente "sus respuestas en las escalas de metacognición y apoyo al procesamiento" de las ACRA ( $r$ ) (Escalas de estrategias de aprendizaje). Y otro descriptivo-correlacional que analizaba la relación entre estrategias metacognitivas y de apoyo al procesamiento y las respuestas de aprendizaje. Los resultados indican diferencias en la auto-percepción que tienen los estudiantes acerca de la adquisición de competencias declarativas y procedimentales después del entrenamiento en autorregulación. La mayor efectividad se observa en el primer curso. Lo que aconseja una metodología de enseñanza-aprendizaje universitaria basada en estrategias de autorregulación.

\section{Palabras clave}

Conocimiento declarativo; conocimiento procedimental; conocimiento metacognitivo; autorregulación. 


\title{
Relationship between academic performance and development of self-regulation strategies in universities students
}

\begin{abstract}
We study the relationship between student's self-perceptions of their declarative and procedural knowledge and their academic performance. 116 university students, in their first and second year of their degree courses participated in two studies. One was quasiexperimental (before-after-no control group), in which the independent variable was "learning based on self-regulation strategies" and the dependent variable was "their responses to scales on metacognition and information processing skills) taken from ACRA (learning strategies scales). A second descriptive-correlational test analyzed the triadic relation between metacognitive strategies, information processing skills and learning outcomes. The results point out to differences in student self-perceptions regarding the acquisition of declarative procedural competencies after self-regulation training. The greatest effectiveness was observed in the first year, lending support to university learningteaching methodology based on self-regulation strategies.
\end{abstract}

\section{Key words}

Declarative knowledge; procedural knowledge; metacognitive knowledge; self-regulation.

\section{Introducción}

Los estudios sobre metacognición tienen dos referentes claros. Por un lado, los trabajos de Flavell (1979) relacionan la metacognición con el conocimiento declarativo y procedimental, y por otro los estudios de Brown y DeLoache (1978) relacionan la metacognición y autorregulación con el logro de aprendizajes eficaces (Castañeda y Villalta, 2017; Cerezo, Fernández, Amieiro, Valle, Rosário, y Núñez, 2019; Rosário, Lourenço, Paiva, Núñez, GonzálezPienda, y Valle, 2012; Veenman, 2007; Veenman, 2011a; Veenman, 2015). Por otra parte, Zimmerman (2008) y Zimmerman y Schunk (2008) consideran que la autorregulación depende de factores socio-emocionales que condicionan el desarrollo metacognitivo. Asimismo, otras investigaciones (Veenman, 2011a,b) indican que la autorregulación se relaciona directamente con el conocimiento procedimental y que éste posee un mecanismo de feedback que ayuda a la construcción del conocimiento (Norman y Furnes, 2016). Dicho conocimiento implica las siguientes estrategias metacognitivas (Van der Stel y Veenman, 2014):

a) Orientación (guía el proceso de resolución y activa los conocimientos previos necesarios).

b) Planificación (permite la secuenciación en pasos del proceso de resolución).

c) Evaluación (evalúa los pasos dados analizando si son efectivos o no para, en su caso, reorientar la planificación).

d) Elaboración (relaciona las conclusiones encontradas con las cuestiones planteadas en el problema o tarea a resolver).

En esta línea los estudios de Román y Gallego (1994) y Román y Poggioli (2013) diferencian dentro de las estrategias metacognitivas, las estrategias de autoconocimiento, planificación 
y evaluación. En la Tabla 1 se puede consultar la relación entre la clasificación de Veenman (2011b) y la de Román y Poggioli (2013).

Tabla 1.

Clasificación de las estrategias metacognitivas de Veenman (2007; 2011b) y Román y Poggioli (2013).

\begin{tabular}{|c|c|c|}
\hline Teoría & $\begin{array}{c}\text { Habilidades metacognitivas } \\
\text { declarativas }\end{array}$ & $\begin{array}{l}\text { Habilidades metacognitivas } \\
\text { procedimentales }\end{array}$ \\
\hline Veeman (2007) & $\begin{array}{l}\text { Relacionadas con la historia de } \\
\text { aprendizaje }\end{array}$ & $\begin{array}{l}\text { Orientación } \\
\text { Planificación } \\
\text { Evaluación } \\
\text { Elaboración }\end{array}$ \\
\hline Román y & Autocontrol & Autoconocimiento \\
\hline Poggioli (2013) & $\begin{array}{l}\text { Contradistractoras } \\
\text { Interacciones sociales } \\
\text { Motivación intrínseca y extrínseca } \\
\text { Motivación de Escape }\end{array}$ & $\begin{array}{l}\text { Planificación } \\
\text { Evaluación }\end{array}$ \\
\hline
\end{tabular}

Las habilidades metacognitivas facilitan el desarrollo de la autorregulación en el proceso de aprendizaje (Meijer, Veenman, y Van Hout-Wolters, 2006; Sáiz y Pérez, 2016). Se observa como aquellos estudiantes que son entrenados en aprendizaje autorregulado mejoran su rendimiento académico y demuestran un mayor compromiso con la realización de tareas (Broadbent y Poon, 2015). Además, estos perfeccionan sus habilidades de planificación, control y regulación de sus actividades académicas (Ventura, Cattoni y Borgobello, 2017). Por otro lado, el modelo predictivo de Gómez y Romero (2019) avala que la autorregulación es la variable que mayor varianza del rendimiento académico explica con un $15.7 \%$. Asimismo, las estrategias metacognitivas son importantes predictores del aprendizaje eficaz (Sáiz, Montero, Bol, y Carbonero, 2012; Van der Stel y Veenman, 2014) ya que explican el 40\% de la varianza en los resultados de aprendizaje (Veenman, 2011b). Igualmente Ventura et al. (2017, p.15) señalan que "ser capaz de autorregular adecuadamente el propio aprendizaje impacta positivamente en el rendimiento académico".

El desarrollo de estrategias de autorregulación es imprescindible para guiar los procesos de enseñanza dentro de los contextos de aprendizaje que incluyen sistemáticamente, y cada vez con mayor frecuencia, las nuevas tecnologías (Cerezo, Sánchez-Santillán, Paule-Ruiz, y Núñez, 2016; Järvelä, Malmberg, y Koivuniemi, 2016). El alumnado universitario autorregula su aprendizaje con múltiples acciones haciendo uso de las tecnologías (Sáiz, Queiruga, GarcíaOsorio, Montero, y Rodríguez-Medina, 2019; Chaves, Trujillo, y López, 2016; Gros y GarcíaPeñalvo, 2016; Marcelo y Rijo, 2019). Desde esta premisa, los entornos virtuales facilitan el entrenamiento de estrategias de autorregulación, lo cual según Díaz, Pérez, González-Pienda y Núñez (2017) tiene un impacto favorable sobre el aprendizaje autorregulado. Por otro lado, se observa que el uso de entornos virtuales de aprendizaje y evaluación inciden de forma significativa en el desarrollo del aprendizaje autorregulado en el ámbito universitario (Castro, Suárez y Soto, 2016; Martínez-Sarmiento y Gaeta, 2019).

Además, en este complejo entramado de conceptos alrededor del término de metacognición se plantea el problema de la medición o evaluación de las estrategias metacognitivas. Los trabajos de Veenman (2011a y 2011b) señalan dos procedimientos para poder efectuarlo. Por un lado, estarían los métodos off-line. Estos métodos hacen referencia a la evaluación a 
través de cuestionarios y entrevistas que se administran al aprendiz antes o después de la resolución de un problema o tarea. Las preguntas se centran en la frecuencia de utilización de las estrategias procedimentales. Y por otro lado, se pueden emplear los métodos on-line, en éstos la evaluación se produce durante la resolución de las tareas o problemas. Dichos métodos utilizan técnicas de observación, protocolos de pensar en voz alta o registros de log-line en el ordenador (Sáiz et al., 2019).

Los métodos off-line tienen la ventaja de que pueden recoger información de muestras amplias de sujetos. Por esta razón se pueden obtener indicadores de fiabilidad y de validez muy altos. Si bien, tienen la desventaja de que la información que proporcionan es la que los aprendices tienen en su memoria sobre cómo resuelven tareas, por lo que dicha información puede estar sujeta a distorsiones (Sáiz y Payo, 2012; Sáiz y Román, 2011; Veenman, 2011b).

Asimismo, los métodos on-line permiten extraer información significativa sobre los procesos de resolución. No obstante, dicha información se recoge de forma individual, lo que dificulta que las muestras sean amplias y como consecuencia los indicadores de fiabilidad y de validez no sean altos (Veenman y van Cleef, 2019). Es por lo que tradicionalmente la evaluación de las estrategias metacognitivas en Educación Superior se ha efectuado utilizando métodos off-line (De Juanas y Beltrán, 2012). Los resultados obtenidos con estos métodos (Rosário, Mourao, Núñez, González-Pienda, Solano, y Valle, 2007; Sáiz y Payo, 2012) indican que los estudiantes universitarios utilizan distintos tipos de estrategias de aprendizaje en función de las titulaciones que cursan. Es decir, respecto del tipo de contenidos que tienen que procesar. Los estudiantes de carreras de ciencias sociales utilizan más las estrategias de autocontrol, planificación y contradistractoras que los de carreras técnicas. Así mismo, las estrategias de motivación de escape y de tolerancia a la frustración parecen estar más desarrolladas en los cursos superiores frente a los iniciales. Por lo que se puede concluir que los procesos de aprendizaje dependen del contexto de aprendizaje y del tipo de tarea (Veenman, Prins, y Verheij, 2003). Un resumen de todo lo reseñado se puede consultar en las Tablas 2 y 3.

Tabla 2.

Relación entre los distintos tipos de metacognición y métodos de evaluación off-line.

\begin{tabular}{|c|c|c|}
\hline & Tipo de metacognición & Evaluación \\
\hline Conocimiento & Conocimiento metacognitivo & Métodos off-line \\
\hline \multirow[t]{4}{*}{ declarativo } & Conocimiento cognitivo & Métodos off-line \\
\hline & $\begin{array}{l}\text { Experiencias metacognitivas (Efklides, 2009; } \\
\text { Flavell, 1979) }\end{array}$ & Métodos off-line \\
\hline & Autoconocimiento & Métodos off-line \\
\hline & Conocimiento condicional & Métodos off-line \\
\hline Conocimiento & Autorregulación de la cognición & Métodos on-line \\
\hline procedimental & Habilidades metacognitivas & Métodos off-line \\
\hline
\end{tabular}

Tabla 3.

Indicadores de evaluación de las habilidades metacognitivas en métodos de evaluación on-line y off-line.

\begin{tabular}{ccc}
\hline $\begin{array}{c}\text { Métodos de evaluación de las } \\
\text { habilidades metacognitivas }\end{array}$ & $\begin{array}{c}\text { Criterios de } \\
\text { evaluación }\end{array}$ & Indicadores de evaluación \\
\hline
\end{tabular}




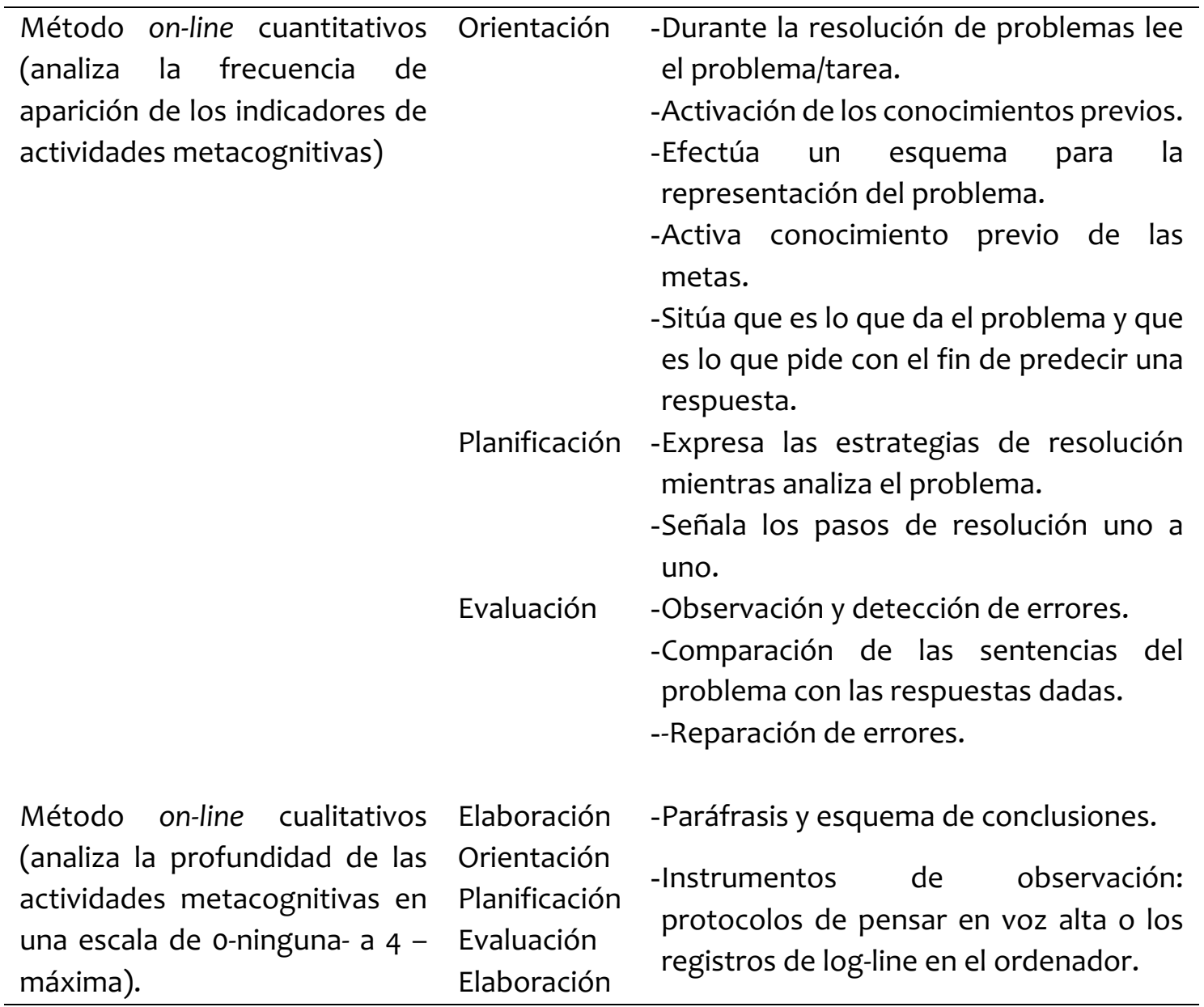

De otro lado hay que considerar que las estrategias metacognitivas se pueden entrenar (Nocito y Navarro, 2018; Schraw y Gutierrez, 2015). En concreto Veenman (2011a) diferencia los siguientes pasos en la instrucción:

a) Posición de síntesis, la instrucción debe de realizarse en el contexto de la tarea. El docente tiene que relacionar las estrategias metacognitivas con las demandas de la tarea a resolver (conocimiento procedimental).

b) Informar de la instrucción, el docente tiene que informar a los estudiantes de los beneficios de aplicar las estrategias metacognitivas. Ya que al inicio del entrenamiento el uso de estas estrategias va a exigir a los estudiantes un mayor esfuerzo de análisis de los procesos de ejecución.

c) Instrucción prolongada, los efectos de la intervención metacognitiva no son significativos en cortos periodos de tiempo. Veenman (2011a) propuso el método WWW\&H (What, When, Why and How). Donde qué (What) implica qué estrategias trabajar, cuándo (When) refiere a cuándo utilizarlas, por qué (Why) implica porque utilizarlas y cómo (How) hace referencia a cómo utilizarlas en contextos de resolución de problemas.

En Educación Superior se han desarrollado experiencias de entrenamiento en estrategias metacognitivas con buenos resultados (Herington y Weaven, 2008; Núñez et al., 2011; Román y Poggioli, 2013; Rosário et al., 2007), ya que este tipo de entrenamiento facilita la activación 
de los conocimientos previos y el desarrollo de aprendizajes significativos (Efklides, 2009; Meijer et al., 2006; Tarricone, 2011).

Orientados y guiados por estos planteamientos teóricos, los objetivos de este estudio fueron (a) comprobar si existían diferencias significativas, antes-después de la instrucción en autorregulación, en la autopercepción que los estudiantes universitarios tienen de sus estrategias metacognitivas procedimentales y (b) comprobar si existían diferencias significativas, antes-después de la instrucción en autorregulación, en la autopercepción de los estudiantes universitarios respecto a sus estrategias metacognitivas declarativas.

Derivadas de estos objetivos, se plantearon cinco hipótesis. Dos de ellas presuponen relaciones funcionales causales:

a) Los sujetos entrenados con un procedimiento metacognitivo-autorregulación mejorarán la percepción de su conocimiento procedimental.

b) Los sujetos entrenados con un procedimiento metacognitivo-autorregulación mejorarán la percepción de su conocimiento declarativo.

Las otras tres hipótesis presuponen relaciones funcionales concomitantes o correlaciónales:

a) Las estrategias metacognitivas procedimentales se relacionarán con los resultados de aprendizaje.

b) Las estrategias metacognitivas declarativas se relacionarán con los resultados de aprendizaje.

c) Las estrategias metacognitivas procedimentales y declarativas no correlacionarán entre sí.

\section{Metodología}

\subsection{Participantes}

Se trabajó con una muestra no probabilística, de tipo incidental o por conveniencia formada por 116 estudiantes $\left(M_{\text {edad }}=22.34, D T_{\text {edad }}=3.99\right)$ de la rama de Ciencias Sociales (62 estudiantes segundo de Grado: 59 mujeres $\left(M_{\text {edad }}=22.5, D T_{\text {edad }}=4.13\right)$ y 3 hombres $\left(M_{\text {edad }}=\right.$ 21.00, $\left.D T_{\text {edad }}=2.1\right)$; 54 estudiantes de primero de Grado: 49 mujeres $\left(M_{\text {edad }}=22.12, D T_{\text {edad }}=\right.$ 7.62) y 5 hombres $\left(M_{\text {edad }}=25.6, D T_{\text {edad }}=13.24\right)$.

\subsection{Diseño}

Para validar las dos primeras hipótesis se utilizó un diseño cuasi-experimental antes-después sin grupo control (Campbell y Stanley, 2005). La variable independiente fue el entrenamiento en autorregulación y se aplicó en ambos grados. La variable dependiente fueron las respuestas a las escalas de metacognición y apoyo al procesamiento de las escalas de estrategias de aprendizaje (ACRA ( $r$ ) ) Román y Poggioli (2013) y la covariable el curso académico (primero vs. segundo). Para la validación de las tres hipótesis restantes se utilizó un diseño descriptivo-correlacional sobre una variable no manipulada experimentalmente (resultados de aprendizaje). 


\subsection{Instrumentos}

a) ACRA (r) - Escala de estrategias de aprendizaje de Román y Poggioli (2013). Las escalas ACRA ( $r$ ) son escalas validadas para población española y sub-americana e identifican 32 estrategias de aprendizaje útiles para optimizar distintos momentos de procesamiento de información: adquisición (estrategias atencionales y de repaso), codificación (nemotecnias, organización y elaboración), recuperación (búsqueda y generación de respuesta), metacognición (autoconocimiento, planificación y regulación y evaluación) y de apoyo al procesamiento (autoinstrucciones, autocontrol, contradistractoras, interacciones sociales, motivación intrínseca y extrínseca y motivación de escape). La fiabilidad del instrumento es adquisición $\alpha=.78$; codificación $\alpha=$.92; recuperación $\alpha=.83$; metacognición $\alpha=.90$ y apoyo al procesamiento $\alpha=.90$. En este estudio se utilizaron las subescalas de metacognición y apoyo al procesamiento, hallándose indicadores de fiabilidad de $\alpha=.89$ y $\alpha=.87$, respectivamente.

b) Resultados de aprendizaje. Estos se obtuvieron mediante un proceso de evaluación continua a lo largo del semestre (en las asignaturas de Psicología) y donde se obtuvo una media ponderada. En dicho proceso se evaluaban los resultados de aprendizaje en las prácticas, el trabajo de investigación, la prueba escrita y coevaluación. La ponderación de cada prueba fue de $20 \%, 40 \%, 30 \%$ y $10 \%$ respectivamente.

\subsection{Procedimiento}

Previo al inicio de la investigación se obtuvo la aprobación del Comité de Bioética de la Universidad de Burgos. Seguidamente, se informó a los estudiantes participantes sobre los objetivos del estudio y se recabó su consentimiento informado por escrito. Posteriormente, se aplicó a todos los estudiantes las escalas de estrategias metacognitivas y de apoyo al procesamiento de las escalas ACRA (r) (Román y Poggioli, 2013). A continuación, se implementó en los dos grupos de estudiantes (primero y segundo de grado) una metodología de enseñanza basada en la autorregulación del aprendizaje ( $S R L$ ) que se efectuó a través de preguntas de indagación apoyadas en imágenes que se aplicaban en las presentaciones en power point. Además, a lo largo del curso académico se recogieron las evidencias de la evaluación continua en ambos grupos y se obtuvo la media ponderada de las calificaciones en la siguiente relación: $30 \%$ pruebas tipo test, $20 \%$ prácticas, $40 \%$ proyecto, y $10 \%$ coevaluación. Asimismo, al finalizar la asignatura se volvió a aplicar en ambos grupos las escalas de estrategias metacognitivas y de apoyo al procesamiento de las escalas ACRA ( $r$ ) (Román y Poggioli, 2013).

\subsection{Análisis de datos}

Para contrastar la primera hipótesis se utilizó un ANOVA de un factor de efectos fijos (procedimiento de intervención metacognitivo-autorregulación). De igual modo, para comprobar la segunda hipótesis se empleó un ANCOVA de un factor de efectos fijos (procedimiento de intervención metacognitivo-autorregulación) y covariable curso académico. Asimismo, para contrastar las hipótesis tercera, cuarta y quinta se utilizó una matriz de correlaciones.

\section{Resultados}

Respecto de la primera hipótesis ("los sujetos entrenados con un procedimiento metacognitivo-autorregulación mejorarán la percepción de su conocimiento procedimental”) se encontraron diferencias significativas antes-después de la intervención 
metacognitiva autorregulada en las estrategias metacognitivas de evaluación $[F(1,115)=3.72$, $\left.p=.05, \eta^{2}=.03\right]$. Asimismo, se apreció el efecto de la covariable: curso académico sobre las estrategias metacognitivas de autoconocimiento $\left[F(1,115)=4.93, p<.05, \eta^{2}=.04\right]$. Asimismo, la varianza total explicada por las estrategias metacognitivas fue de un $3 \%$ ) (ver Tabla 4).

Tabla 4

Comparación de los resultados antes-después de la intervención en la subescala de habilidades metacognitivas de la escala de estrategias metacognitivas de Román y Poggioli (2013) (ANOVA) y análisis del valor del efecto.

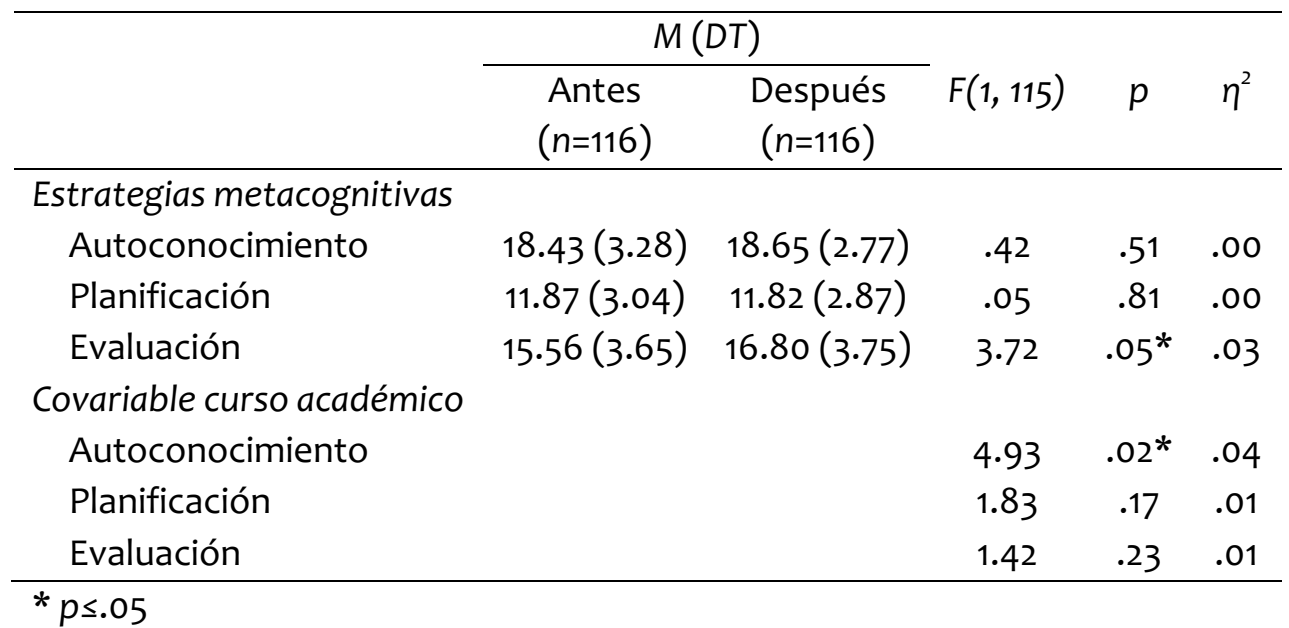

En relación a la segunda hipótesis ("los sujetos entrenados con un procedimiento metacognitivo-autorregulación mejorarán la percepción de su conocimiento declarativo"). Se encontraron diferencias significativas en las estrategias contradistractoras $[F(1,115)=$ $\left.6.29, p \leq .01, \eta^{2}=.05\right]$, las estrategias de motivación intrínseca y extrínseca $[F(1,115)=5.49$, $\left.p \leq .02, \eta^{2}=.04\right]$ y en las estrategias de motivación de escape $\left[F(1,115)=4.45, p \leq .03, \eta^{2}=.03\right]$. En conjunto las estrategias de apoyo al procesamiento explican el $14 \%$ de la varianza. Asimismo, se apreciaron efectos de la covariable en las estrategias de autoinstrucciones $(p \leq .001)$ aunque no se detectaron diferencias significativas en la comparación inter-grupos $(p \leq .12)$ (ver Tabla 5).

Tabla 5

Comparación de los resultados antes-después de la intervención en la subescala de habilidades de apoyo al procesamiento de la escala de estrategias metacognitivas de Román y Poggioli (2013) (ANcOVA) y análisis del valor del efecto.

\begin{tabular}{lccccc}
\hline & \multicolumn{2}{c}{$M(D T)$} & & & \\
\cline { 2 - 4 } & $\begin{array}{c}\text { Antes } \\
(n=116)\end{array}$ & $\begin{array}{c}\text { Después } \\
(n=116)\end{array}$ & $F(1,115)$ & $p$ & $\eta^{2}$ \\
\hline Estrategias de apoyo al procesamiento & & & & & \\
$\quad$ Autoinstrucciones & $14.71(2.60)$ & $14.65(3.29)$ & 2.37 & .12 & .02 \\
$\quad$ Autocontrol & $2.53(1.06)$ & $2.42(.95)$ & .38 & .53 & .00
\end{tabular}




\begin{tabular}{lccccc} 
Contradistractoras & $8.12(1.76)$ & $8.35(2.34)$ & 6.29 & $.01 *$ & .05 \\
Interacciones sociales & $11.98(2.34)$ & $11.45(2.65)$ & .12 & .72 & .00 \\
Motivación intrínseca y extrínseca & $10.67(2.10)$ & $10.29(3.14)$ & 5.49 & $.02 *$ & .04 \\
Motivación de escape & $2.24(1.03)$ & $2.55(1.03)$ & 4.45 & $.03 *$ & .03 \\
Covariable curso académico & & & & & \\
$\quad$ Autoinstrucciones & & & 82.86 & $.00 *$ & .43 \\
Autocontrol & & .11 & .73 & .00 \\
Contradistractoras & & .51 & .47 & .00 \\
Interacciones sociales & & & .72 & .39 & .00 \\
Motivación interna y externa & & & 1.77 & .18 & .01 \\
Motivación de escape & & & .01 & .89 & .00 \\
\hline$p \leq .05$ & & & & &
\end{tabular}

Referente a la tercera hipótesis ("las estrategias metacognitivas procedimentales se relacionarán con los resultados de aprendizaje”) no se encontraron correlaciones significativas entre las calificaciones obtenidas por los sujetos en las habilidades procedimentales después de la intervención (ver Tabla 6).

Tabla 6

Correlaciones entre las estrategias metacognitivas (Román y Poggioli, 2013) y las calificaciones obtenidas por los sujetos en las materias.

\begin{tabular}{|c|c|c|c|c|c|}
\hline & 1 & 2 & 3 & 4 & 5 \\
\hline 1. Estrategias metacognitivas de autoconocimiento & 1 & & & & \\
\hline 2. Estrategias metacognitivas de planificación & .00 & 1 & & & \\
\hline 3. Estrategias metacognitivas de evaluación & $.54^{* *}$ & .00 & 1 & & \\
\hline 4. Total Habilidades metacognitivas & $.72^{* *}$ & $.56^{* *}$ & $.86^{* *}$ & 1 & \\
\hline 5. Resultados de aprendizaje & .10 & .01 & -.07 & .01 & 1 \\
\hline Media & 18.65 & 11.82 & 16.80 & 46.27 & 7.36 \\
\hline Desviación típica & 2.77 & 2.87 & 3.75 & 3.13 & .93 \\
\hline
\end{tabular}

Cuarta hipótesis ("las estrategias metacognitivas declarativas se relacionarán con los resultados de aprendizaje”). No se encontraron correlaciones significativas en habilidades de apoyo al procesamiento (conocimiento declarativo) y los resultados de aprendizaje después de la intervención (ver Tabla 7).

Quinta hipótesis ("las estrategias metacognitivas procedimentales y declarativas no correlacionarán entre si"). Como puede observarse en la Tabla 8 las correlaciones entre las puntuaciones totales en estrategias metacognitivas (habilidades procedimentales) y de apoyo al procesamiento (habilidades declarativas) no correlacionan de forma significativa siendo además el signo de correlación negativo $(r=-.11)$. Tampoco se aprecian correlaciones significativas entre las habilidades que conforman cada una de las escalas: autoconocimiento y autoinstrucciones $(r=-.22)$; autoconocimiento y contradistractoras $(r=-.14)$; 
autoconocimiento e interacciones sociales $(r=-.14)$; autoconocimiento y motivación intrínseca y extrínseca $(r=-.20)$, autoconocimiento y motivación de escape $(r=-.16)$. planificación y autoinstrucciones $(r=.09)$; planificación y contradistractoras $(r=-.09)$; planificación e interacciones Sociales $(r=.08)$; planificación y motivación intrínseca y extrínseca $(r=.12)$, planificación y motivación de escape $(r=-.05)$; evaluación y autoinstrucciones $(r=-.17)$; evaluación y contradistractoras $(r=-.01)$; evaluación e interacciones Sociales $(r=-.11)$; evaluación y motivación intrínseca y extrínseca $(r=.01)$, evaluación y motivación de escape $(r=-.09)$ (ver Tabla 8 ).

Tabla 7

Correlaciones entre las habilidades de la escala de apoyo al procesamiento de Román y Poggioli (2013) y las calificaciones obtenidas por los sujetos en las materias.

\begin{tabular}{|c|c|c|c|c|c|c|c|c|}
\hline & 1 & 2 & 3 & 4 & 5 & 6 & 7 & 8 \\
\hline $\begin{array}{l}\text { 1. Estrategias de } \\
\text { autoinstrucciones }\end{array}$ & 1 & & & & & & & \\
\hline 2. Estrategias de autocontrol & $.47^{* *}$ & 1 & & & & & & \\
\hline 3. Estrategias contradistractoras & $.45^{* *}$ & $.27^{*}$ & 1 & & & & & \\
\hline $\begin{array}{l}\text { 4. Estrategias de interacciones } \\
\text { sociales }\end{array}$ & $.68^{* *}$ & $.25^{*}$ & $.51^{* *}$ & 1 & & & & \\
\hline $\begin{array}{l}\text { 5. Estrategias de motivación } \\
\text { intrínseca y extrínseca }\end{array}$ & $.54^{* *}$ & $.32 *$ & $.44^{* *}$ & $.58^{* *}$ & 1 & & & \\
\hline $\begin{array}{l}\text { 6. Estrategias de motivación de } \\
\text { escape }\end{array}$ & .22 & .12 & $.26^{*}$ & $.28^{*}$ & $.41^{* *}$ & 1 & & \\
\hline $\begin{array}{l}\text { 7. Total habilidades de apoyo al } \\
\text { procesamiento }\end{array}$ & $.77 * *$ & $.83^{* *}$ & $.51^{* *}$ & $.82^{* *}$ & $.85^{* *}$ & $.48^{* *}$ & 1 & \\
\hline 8. Resultados de aprendizaje & .12 & .00 & -.23 & .20 & .11 & -.02 & .12 & 1 \\
\hline Media & 16.65 & 2.42 & 8.35 & 11.45 & 14.62 & 2.55 & 41.42 & 7.36 \\
\hline Desviación Típica & 3.29 & .95 & 2.34 & 2.65 & 3.56 & 1.03 & 1.71 & .93 \\
\hline
\end{tabular}

Tabla 8

Correlaciones entre las habilidades de la escala de apoyo al procesamiento de Román y Poggioli (2013) y las calificaciones obtenidas por los sujetos en las materias.

\begin{tabular}{|c|c|c|c|c|c|c|c|c|c|c|c|}
\hline & 1 & 2 & 3 & 4 & 5 & 6 & 7 & 8 & 9 & 10 & 11 \\
\hline $\begin{array}{l}\text { Estrategias metacognitivas de } \\
\text { autoconocimiento }\end{array}$ & 1 & & & & & & & & & & \\
\hline $\begin{array}{l}\text { Estrategias metacognitivas de } \\
\text { planificación }\end{array}$ & .00 & 1 & & & & & & & & & \\
\hline $\begin{array}{l}\text { Estrategias metacognitivas de } \\
\text { evaluación }\end{array}$ & $.54^{* *}$ & $.28^{*}$ & 1 & & & & & & & & \\
\hline Total habilidades metacognitivas & $.72^{* *}$ & $.56^{* *}$ & $86^{* *}$ & 1 & & & & & & & \\
\hline
\end{tabular}




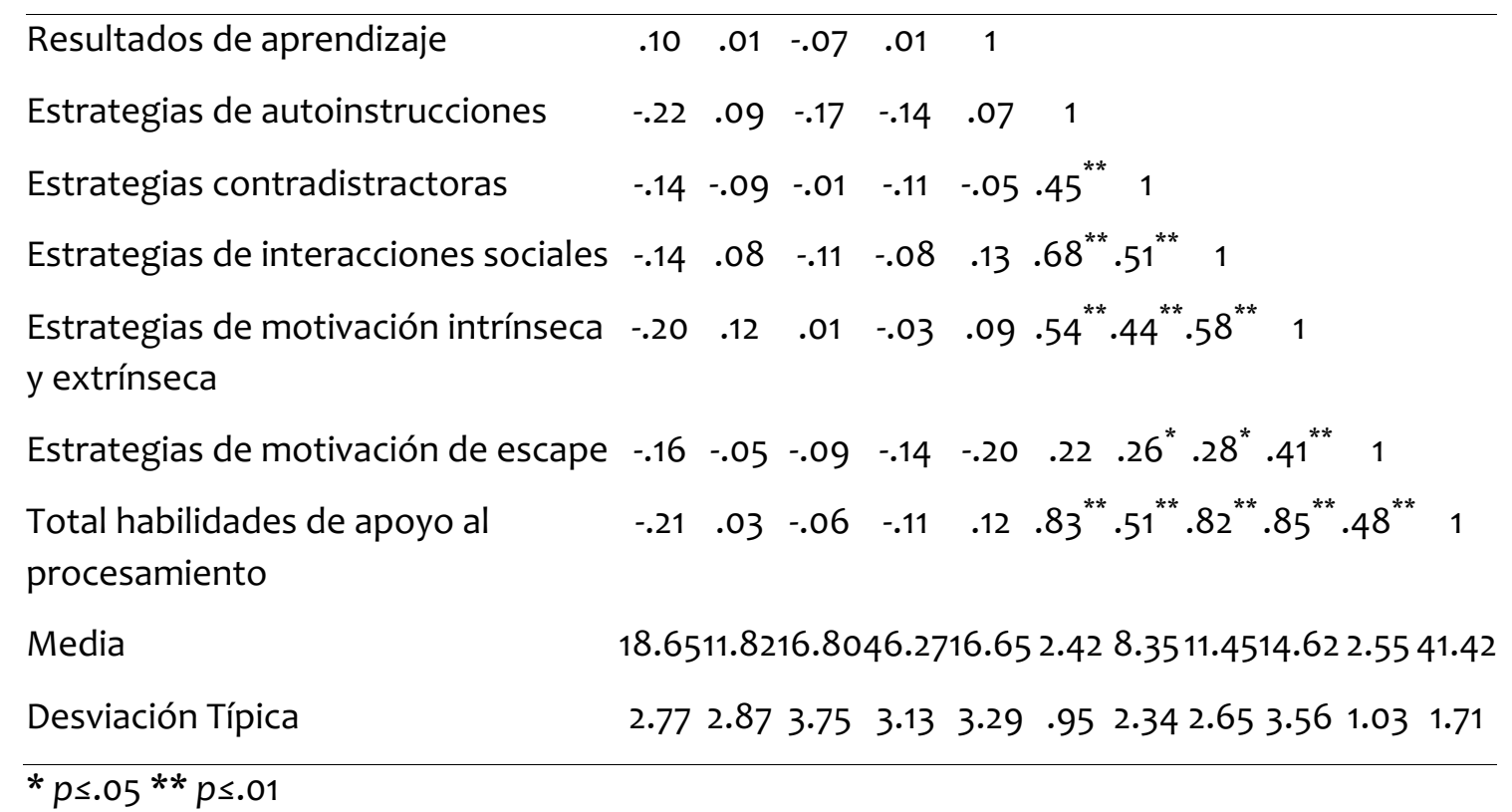

\section{Discusión}

El entrenamiento en aprendizaje autorregulado (SRL) parece tener los mismos efectos sobre las estrategias declarativas que sobre las procedimentales. En éstas últimas los cambios significativos se relacionan con las estrategias metacognitivas de evaluación, pero no con las de autoconocimiento ni con las de planificación. Por lo que se podría inferir que este tipo de entrenamiento parece tener más efectos sobre el conocimiento declarativo [estrategias de apoyo al procesamiento, como son las estrategias contradistractoras, las de motivación interna y externa y las de motivación de escape (tolerancia a la frustración)] que sobre el procedimental (estrategias de autoconocimiento y planificación). Estos resultados coinciden con los hallados por Veenman (2011a) relativos a la importancia de la autopercepción del estudiante sobre su conocimiento declarativo (Sáiz y Payo, 2012; Sáiz et al., 2012) para la consecución de aprendizajes eficaces basados en la motivación de logro en estudiantes universitarios. Es decir, la percepción del conocimiento metacognitivo por parte del alumnado universitario parece estar vinculada a su historia de aprendizaje. Por lo que estos resultados respaldan la tesis sobre la diferencia en la adquisición de las estrategias procedimentales y declarativas (Veenman, 2011a; 2011b). Las primeras están vinculadas con el cómo orientar la resolución de tareas y de problemas y tienen que ver con procesos ejecutivos de orden superior, por lo que quizás no puedan ser entrenadas de forma efectiva en periodos cortos de tiempo (Veenman, 2011b). Las segundas se relacionan con la motivación y esta parece que es más susceptible de cambio en periodos de entrenamiento más cortos (Veenman, 2011a).

En esta línea, los resultados encontrados de ausencia de correlaciones significativas y en ocasiones negativas entre las estrategias metacognitivas (procedimentales) y las de apoyo al procesamiento (declarativas) apoyan también la tesis de Veenman (2011a, 2011b) sobre la diferencia en el origen entre el conocimiento declarativo y el procedimental.

Asimismo, la autopercepción de las estrategias metacognitivas no parece tener relación con los resultados de aprendizaje. Lo que apoya la tesis de Veenman (2011a; 2011b) y 
colaboradores (Veenman y van Cleef, 2019) sobre la dificultad de evaluar dichas estrategias a través de métodos off-line. Por ello, futuras investigaciones irán dirigidas a contrastar estos resultados con los obtenidos con técnicas de evaluación on-line (protocolos de pensar en voz alta).

Respecto de la relación entre las estrategias de apoyo al procesamiento (declarativas) y los resultados de aprendizaje de los alumnos se han hallado relaciones significativas con las estrategias de autocontrol. Si bien, estas son negativas lo que lleva a plantearse que quizás la autopercepción que el aprendiz tiene sobre sus propias estrategias de autocontrol del aprendizaje puede estar influida por su historia de aprendizaje lo que también apoyaría la tesis de Veenman (2011a).

Igualmente, se encontró relación entre los resultados de aprendizaje y las estrategias de motivación (Veenman, 2011a, b). Este resultado apoyaría la hipótesis de Zimmerman (2008) sobre la importancia de la motivación en los procesos de enseñanza-aprendizaje.

\section{Conclusiones}

En síntesis, parece existir una diferencia en la adquisición de estrategias metacognitivas (procedimentales) y las de apoyo al procesamiento (declarativas). Este es un indicador importante a la hora de diseñar programas instruccionales metacognitivos basados en la autorregulación, ya que es necesario favorecer esta estrategia como parte fundamental del proceso de enseñanza personalizada (Cerezo et al., 2016; Pérez y Solís, 2016; Sáiz y Pérez, 2016; Sáiz et al., 2012; Sáiz et al., 2019). Además, la autopercepción que el estudiante tiene de su propio conocimiento parece no correlacionar con los resultados de aprendizaje (Sáiz y Payo, 2012). Este es un aspecto importante a trabajar en el marco de la Educación Superior, ya que una percepción ajustada de los conocimientos y habilidades es necesaria para la formación de futuros profesionales. El objetivo último es que adquieran competencias y las desarrollen con eficacia y seguridad.

No obstante, los datos encontrados en este estudio deben tomarse con la prudencia propia hacia los estudios cuasi-experimentales sin grupo control. Por lo que se propone continuar la investigación con estudios longitudinales sobre el entrenamiento metacognitivo basado en SRL en estudiantes universitarios (Veenman, 2011a) introduciendo grupo de control. Además de señalar la importancia de llevar a cabo entrenamientos instruccionales basados en SRL durante el primer año de acceso a los grados universitarios (Nocito y Navarro, 2018; Schraw y Gutierrez, 2015), tanto para el desarrollo del conocimiento declarativo como del procedimental en Educación Superior. Así mismo, hay que tener en cuenta que según Cerezo et al. (2019) el alumnado no utilizará SRL si el esfuerzo invertido es demasiado alto frente a una recompensa que considera insuficiente. Para solucionar este inconveniente, los autores señalan que la utilidad percibida es una variable motivacional fundamental dentro de los procesos de intervención en SRL (Järvelä et al., 2016).

En consecuencia, la implicación y apoyo de los docentes ha demostrado tener un impacto favorable sobre la autorregulación del aprendizaje en los entornos virtuales (Díaz et al., 2017), así como, la necesaria incorporación de las tecnologías de la información y la comunicación dentro del diseño de los propios programas académicos se considera una herramienta que fomenta el aprendizaje autorregulado en el alumnado de Educación Superior (Cerezo et al., 2016).

No obstante, para que todo este proceso instruccional se pueda llevar a efecto se tiene que efectuar de una forma cohesionada y coordinada, es decir la experiencia tiene que ser 
apoyada por los coordinadores de titulación y los decanos o directores de centros. Lo que implica un reto en la organización de las titulaciones y del proceso de enseñanza-aprendizaje en las universidades.

\section{Referencias}

Broadbent, J., y Poon, W. L. (2015). Self-regulated learning strategies andacademic achievement in online higher education learning environ-ments: A systematic review. The Internet and Higher Education, 27, 1-13. doi: 10.1016/j.iheduc.2015.04.007

Brown, A., y DeLoache, J. S. (1978). Skills, Plans and self-regulation. En R. S. Siegel (Ed.), Children's thinking: What develops? (pp. 3-35). Hillsdale, N.J.: Erlbaum.

Campbell, D. T., y Stanley, J. C. (2005). Diseños experimentales y cuasiexperimentales en la investigación social. Buenos Aires: Amorrortu [ $1^{\mathrm{a}}$ edición en castellano 1973; novena reimpresión].

Castañeda, M. T., y Villalta, M. A. (2017). Gestión del aula y formación inicial de profesores. Un estudio de Revisión. Perspectiva educacional, 56(2), 4-27. Recuperado de http://www.perspectivaeducacional.cl/index.php/peducacional/issue/view/37

Castro, N., Suárez, X., y Soto, V. (2016). El uso del foro virtual para desarrollar el aprendizaje autorregulado de los estudiantes universitarios. Innovación Educativa, 16(70), $23-42$. Recuperado de https://www.ipn.mx/innovacion/números-anteriores/innovacióneducativa-70.html

Cerezo, E., Fernández, E., Amieiro, N., Valle, A., Rosário, P., y Núñez, J. C. (2019). El papel mediador de la autoeficacia y la utilidad entre el conocimiento y el uso de estrategias de autorregulación del aprendizaje. Revista de Psicodidáctica, 24(1), 1-8. doi: 10.1016/j.psicod.2018.08.001

Cerezo, R., Sánchez-Santillán, M., Paule-Ruiz, M. P., y Núñez, J. C. (2016). Students' LMS interaction patterns and their relationship with achievement: A case study in higher education. Computers \& Education, 96, 42-54. doi: 10.1016/j.compedu.2016.02.006

Chaves, E., Trujillo, J. M., y López, J. A. (2016). Acciones para la autorregulación del aprendizaje en entornos personales. Píxel-Bit. Revista de Medios y Educación, (48), 6782. doi: 10.12795/pixelbit.2016.i48.05

De Juanas, A., y Beltrán, J. A. (2012). Creencias epistemológicas de los estudiantes de Pedagogía y Ciencias de la Educación. Revista de Psicodidáctica, 17(1), 1-15. Recuperado de http://www.ehu.eus/ojs/index.php/psicodidactica/article/view/1226/1996

Díaz, A., Pérez, M. V., González-Pienda, J. A., y Núñez, J. C. (2017). Impacto de un entrenamiento en aprendizaje autorregulado en estudiantes universitarios. Perfiles Educativos, 39(157), 87-104. Recuperado de http://www.iisue.unam.mx/perfiles/numeros/2017/157

Efklides, A. (2009). The role of metacognitive experiences in the learning process. Psicothema, 21(1), 76-82. Recuperado de http://www.psicothema.com/psicothema.asp?ID=3598

Flavell, J. H. (1979). Metacognition and cognitive monitoring. Americam Psychologist, 34, 906911. doi: 10.1037/0003-066X.34.10.906

Gómez, J., y Romero, A. (2019). Enfoques de aprendizaje, autorregulación y autoeficacia y su influencia en el rendimiento académico en estudiantes universitarios de Psicología. European Journal of Investigation in Health, Psychology and Education, 9(2), 95-107. doi: 10.30552/ejihpe.vgi2.323 
Gros, B., y García-Peñalvo, F. J. (2016). Future Trends in the Design Strategies and Technological Affordances of E-Learning. En J. M. Spector, B. B Lockee y M. D. Childress (Eds.), Learning, Design, and Technology (pp. 1-23). Switzerland: Springer. doi: 10.1007/978-3-319-17727-4_67-1

Herington, C., y Weaven, S. (2008). Action research and reflection on student approaches to learning in large first year university classes. The Australian Educational Researcher, 35(3), 111-134. doi: 10.1007/BF03246292

Järvelä, S., Malmberg, J., y Koivuniemi, M. (2016). Recognizing socially shared regulation by using the temporal sequences of online chat and logs in CSCL. Learning and Instruction, 42, 1-11. doi: 10.1016/j.learninstruc.2015.10.006

Marcelo, C., y Rijo, D. (2019). Aprendizaje autorregulado de estudiantes universitarios: Los usos de las tecnologías digitales. Revista Caribeña de Investigación Educativa (RECIE), 3(1), 62-81. doi: 10.32541/recie.2019.v3i1.pp62-81

Martínez-Sarmiento, L. F., y Gaeta, M. L. (2019). Utilización de la plataforma virtual Moodle para el desarrollo del aprendizaje autorregulado en estudiantes universitarios. Educar, 55(2), 479-498. doi: 10.5565/rev/educar.883

Meijer, J., Veenman, M. V. J., y Van Hout-Wolters, B. H. A. M. (2006). Metacognitive Activities in Text-Studying and Problem-Solving: Development of a taxonomy. Educational Research and Evaluation, 12(3), 209-237. doi: 10.1080/13803610500479991

Nocito, G., y Navarro, E. (2018). Mejora de las estrategias de autorregulación del aprendizaje en la universidad impacto de un programa de adaptación académica a grado. Bordón, Revista de Pedagogía, 70(4), 121-136. doi. 10.13042/Bordon.2018.60148

Norman, E., y Furnes, B. (2016). The relationship between metacognitive experiences and learning: Is there a difference between digital and non-digital study media?. Computers in Human Behavior, 54, 301-309. doi: 10.1016/j.chb.2015.07.043

Núñez, J. C., Cerezo, R., Bernardo, A., Rosario, P., Valle, A., Fernández, E., y Suárez, N. (2011). Implementation of training programs in self-regulated strategies in Moodle format: Results of an experience in higher education. Psicothema, 23(2), 274-281. Recuperado de http://www.psicothema.com/psicothema.asp?ID=3882

Pérez, J., y Solís, S. (2016). La autorregulación en el proceso de enseñanza y aprendizaje: percepción de los estudiantes de la Cátedra de Turismo Sostenible de la UNED. Revista Calidad en la Educación Superior, 7(1), 148-174. doi: 10.22458/caes.v7i1.1380

Román, J. M., y Gallego, S. (1994). Escalas de estrategias de aprendizaje: ACRA. Madrid: TEA.

Román, J. M., y Poggioli, L. (2013). ACRA (r): Escalas de estrategias de aprendizaje. Caracas: Publicaciones UCAB.

Rosário, P., Lourenço, A., Paiva, M. O., Núñez, J. C., González-Pienda, J. A., y Valle, A. (2012). Autoeficacia y utilidad percibida como condiciones necesarias para un aprendizaje académico autorregulado. Anales de psicología, 28(1), 37-44. Recuperado de http://revistas.um.es/analesps/article/view/140502

Rosário, P., Mourao, R., Núñez, J. C., González-Pineda, J., Solano, P., y Valle, A. (2007). Eficacia de un programa instruccional para la mejora de procesos y estrategias de aprendizaje en la enseñanza superior. Psicothema, 19(39), 422-427. Recuperado de http://www.psicothema.com/psicothema.asp?ID $=3380$

Sáiz, M.C., Montero, E., Bol, A., y Carbonero, M.A. (2012). An analysis of Learning Competences at the University. Electronic Journal of Research in Educational Psychology, 10(1), 253-270. Recuperado de http://www.investigacionpsicopedagogica.org/revista/new/index.php?n=26 
Sáiz, M.C., y Payo, R.J. (2012). Autopercepción del conocimiento en Educación Superior. Revista Iberoamericana de Psicología y Salud, 3(2), 159-174. Recuperado de http://www.redalyc.org/articulo.oa?id=245124456005

Sáiz, M.C., y Pérez, M. (2016). Autorregulación y mejora del autoconocimiento en resolución de problemas. Psicología desde el Caribe, 33(1), 14-30. Recuperado de http://www.redalyc.org/articulo.oa?id=21345152002

Sáiz, M.C., Queiruga, M.Á., García-Osorio, C.I., Montero, E., y Rodríguez-Medina, J. (2019). Observation of Metacognitive Skills in Natural Environments: A Longitudinal Study With Mixed Methods. Front. Psychol., 10, 1-13. doi: 10.3389/fpsyg.2019.02398

Sáiz, M.C., y Román, J.M. (2011). Cuatro formas de evaluación en Educación Superior gestionadas desde la tutoría. Revista de Psicodidáctica, 16(1), 145-161. Recuperado de http://www.redalyc.org/articulo.oa?id=17517217008

Schraw, G., y Gutierrez, A. P. (2015). Metacognitive Strategy Instruction that highlights the Role of Monitoring and Control Processes. En A. Peña-Ayala, Metacognition: Fundaments, Applications, and Trends, (Vol. 76 series Intelligent Systems Reference Library, pp. 3-16). Switzerland: Springer. doi: 10.1007/978-3-319-11062-2_1

Tarricone, P. (2011). The Taxonomy of Metacognition. U.K: Psychology Press.

Van der Stel, M., y Veenman, M. V. J. (2014). Metacognitive Skills and Intellectual Ability of Young Adolescents: A Longitudinal Study from a Developmental Perspective. European Journal of Psychology of Education, 29, 117-137. doi: 10.1007/s10212-013-01905

Veenman, M. V. J. (2007). The assessment and instruction of self-regulation in computerbased environments: A discussion. Metacognition and Learning, 2, 177-183. doi: 10.1007/s11409-007-9017-6

Veenman, M. V. J. (2011a). Alternative assessment of strategy use with self-report instruments: a discussion. Metacognition and Learning, 6, 205-211. doi: 10.1007/s11409011-9080-x

Veenman, M. V. J. (2011b). Learning and Self-Monitor and Self-Regulate. En R. Mayer y P. Alexander. (Eds.), Handbook of Research on Learning and Instruction ( $p p$.197-218). New York: Routledge.

Veenman, M. V. J. (2015). Thinking about metacognition improves thinking. En R. Wegerif y J. C. Kaufman (Eds.), The Routledge International Handbook of Research on Teaching Thinking (pp. 280-288). University of Exeter: Taylor and Francis Inc. doi: 10.4324/9781315797021

Veenman, M. V. J., y van Cleef, D. (2019). Measuring metacognitive skills for mathematics: students' self-reports versus on-line assessment methods. ZDM - Mathematics Education, 51(4), 691-701. doi: 10.1007/s11858-018-1006-5

Veenman, M. V. J., Prins, F. J., y Verheij, J. (2003). Learning styles: Self-report versus thinkingaloud measures. British Journal of Educational Psychology, 73(3), 357-372. doi: 10.1348/000709903322275885

Ventura, A. C., Cattoni, M. S., y Borgobello, A. (2017). Aprendizaje autorregulado en el nivel universitario: Un estudio situado con estudiantes de psicopedagogía de diferentes ciclos académicos. Revista electrónica educare, 21(2), 1-20. doi: 10.15359/ree.21-2.15

Zimmerman, B. J. (2008). Investigating self-regulation and motivation: historical background, methodological developments, and future prospects. American Educational Research Journal, 45, 166-183. doi: 10.3102/0002831207312909 
Zimmerman, B. J., y Schunk, D. (2008). Motivation. An essential dimension of self-regulated learning. En D. Schunk y B. J. Zimmerman (Eds.), Motivation and Self-regulated learning. Theory, research and applications (pp. 1-31). New York: Lawrence Erlbaum. 


\section{Anexo}

Preguntas de autorregulación en el entrenamiento metacognitivo en Respuestas autoinstrucciones

\begin{tabular}{l}
\hline Orientación \\
\hline 1. “¿Qué información relevante da el guión de la práctica?”. \\
2. “¿Qué relación existe entre la información relevante y los conocimientos \\
previos que yo tengo que tener para realizar la práctica?”. \\
3. “¿Qué conceptos no comprendo?”. \\
4. “¿Qué información necesito para comprender dichos conceptos?”. \\
5. “¿Qué otras materias están relacionadas con esos conceptos que no \\
tengo?”. \\
6. “¿Qué ayuda debería de pedir al profesor para poder comprender esos \\
conceptos?”. \\
\hline Planificación \\
\hline 7. “¿Qué pasos debo seguir para resolver la práctica y alcanzar los objetivos \\
que me plantea?”. \\
8. “¿Qué hipótesis tengo que plantear para resolver la práctica?”. \\
\hline Evaluación \\
\hline 9. “¿Debo verificar cada una de las hipótesis que me propuse en el plan de \\
resolución?”. \\
10. “¿Debo comparar los resultados finales con las cuestiones que \\
planteaba el guión?”. \\
11. “¿Debo analizar las desviaciones respecto de los resultados previstos y \\
proponer nuevas hipótesis para resolver las divergencias?”. \\
\hline Elaboración \\
\hline 12. “¿Cuál es la información más importante para resolver la práctica?”. \\
13. “¿Cuál es la información menos importante en la resolución de la \\
práctica?”. \\
14. “Tras realizar la práctica ¿en qué tipo de situación de mi futura práctica \\
profesional puedo usar lo que he aprendido?”. \\
\hline
\end{tabular}

